\title{
Astrocytic Glycogen Mobilization in Cerebral Ischemia/Reperfusion Injury
}

\author{
Doutong Yu¹, Yuting Qiao', Haiyun $\mathrm{Guo}^{3}$, Zejun $\mathrm{Gao}^{4}$, Li Guo ${ }^{1}$, Yanhui Cai ${ }^{*}$, Yulong $\mathrm{Ma}^{2 *}$ \\ ${ }^{1}$ Department of Psychiatry, Xijing Hospital, Fourth Military Medical University, Xi'an 710032, Shaanxi, China \\ ${ }^{2}$ Department of Anesthesiology, the First Medical Center of Chinese PLA General Hospital, Beijing 100853, China \\ ${ }^{3}$ Department of Anesthesiology and Perioperative Medicine, Xijing Hospital, Fourth Military Medical University, Xi'an 710032, Shaanxi, China \\ ${ }^{4}$ Department of Otolaryngology-Head and Neck Surgery, Xijing Hospital, Fourth Military Medical University, Xi'an 710032, Shaanxi, China
}

*Corresponding Author: Yanhui Cai, Department of Psychiatry, Xijing Hospital, Fourth Military Medical University, Xi'an 710032, Shaanxi, China and Yulong Ma, Department of Anesthesiology, the First Medical Center of Chinese PLA General Hospital, Beijing 100853, China.

\section{Received date: December 31, 2022; Accepted date: January 28, 2022; Published date: February 03,2022}

Citation: Doutong Yu, Yuting Qiao, Haiyun Guo, Zejun Gao, Li Guo. et all (2022). Astrocytic Glycogen Mobilization in Cerebral Ischemia/Reperfusion Injury. J. Neuroscience and Neurological Surgery. 11(3); DOI:10.31579/2578-8868/228

Copyrights: (c) 2022 Yanhui Cai, Yulong Ma, This is an open-access article distributed under the terms of The Creative Commons Attribution License, which permits unrestricted use, distribution, and reproduction in any medium, provided the original author and source are credited

\begin{abstract}
Glycogen is an important energy reserve in the brain and can be rapidly degraded to maintain metabolic homeostasis during cerebral blood vessel occlusion. Recent studies have pointed out the alterations in glycogen and its underlying mechanism during reperfusion after ischemic stroke. In addition, glycogen metabolism may work as a promising therapeutic target to relieve reperfusion injury. Here, we summarize the progress of glycogen metabolism during reperfusion injury and its corresponding application in patients suffering from ischemic stroke.
\end{abstract}

Key words: ischemia/reperfusion injury; astrocyte; glycogen; neuroprotection

\section{Introduction}

Ischemic stroke is the second leading cause of death in the world, and vascular recanalization by thrombolysis is the main treatment method at present, but thrombolysis can cause reperfusion injury. [1] The main mechanisms of reperfusion injury include glutamate poisoning, excess oxygen free radicals, inflammation and calcium overload. The deficiency and restoration of blood flow during ischemia/reperfusion (I/R) can disrupt energy balance in the brain, which can aggravate the disruption of brain homeostasis. Accordingly, it is crucial to uncover the alterations in energy metabolism and metabolic disorders during I/R.

In the central nervous system, astrocytes account for $20 \%-24 \%$ of the total number of cells, and their number even exceeds that during neurological diseases, such as ischemia. [2] As a prior target for the neuroprotection of ischemic stroke, astrocytes contact, interact with and affect all parenchymal cells. [3, 4] Cerebral ischemia affects all cellular components in the brain and initiates signaling responses between similar and different types of cells. [5]

Astrocytes play important roles in the brain, including neurotransmitter absorption and secretion, and they maintain the blood-brain barrier by providing biochemical support to the endothelial cells of the blood-brain barrier. Astrocytes provide nutrients to the nervous tissues to maintain the balance of extracellular ions and play a role in the repair processes following head and spinal cord trauma and scar formation. [6] Glycogen is found mainly in astrocytes but not in neurons. When the brain is ischemic, astrocytes located near neurons in the frontal cortex and hippocampus supply these neurons with glucose. [7] Glycogen, a high polymer of glucose, accumulates in astrocytes during ischemia-reperfusion, mainly because of impaired glycogen decomposition. Based on these findings, there is a protective effect of glycogen decomposition on neurons. [8] This project mainly discusses the role and mechanism of astrocyte glycogen metabolism in cerebral ischemia-reperfusion injury.

\section{Glycogen alterations during I/R}

Glycogen is the stored form of glucose in cells. Glycogen is a high molecular compound of glucose and is the second largest energy reserve in the human body behind fat, which mainly exists in astrocytes. Brain glycogen, as an important endogenous energy substance in the brain, mainly exists in astrocytes and is rapidly decomposed to provide additional energy support for the brain when blood vessels are blocked. [9] As an important energy source for astrocytes, glycogen is a regulator of astrocyte utilization of blood-borne glucose. [10, 11] During brain stimulation, glycogen decomposition could meet the energy requirements by inhibiting astrocyte hexokinase and glycogen decomposition promotes glutamate resynthesis in astrocytes by astrocyte utilization of extracellular and blood-derived grapes. $[10,12]$ Pyruvate is produced by astrocyte glycogen and can achieve aerobic metabolism by traveling to neurons and providing energy for neuronal 
plasticity. Meanwhile, the dehydration of the glycogen carbon can be produced through the carboxylation of pyruvate to produce glutamate. [1316]

Ischemic stroke is a disease with a high incidence and fatality rate. Because of the reduced blood flow and lowered oxygen and glucose levels in the brain, cerebral ischemia causes neuronal injury or death in part of the brain, and the common cause of damage is hemisphere contralateral hemiplegia. Ischemic stroke causes problems for patients. For example, patients have difficulty moving without assistance. Hundreds of patients cannot spend their time as they wish, and some patients even suffer from permanent disease. [17-19]

Cerebral ischemia affects all cellular components of the brain and induces signaling responses between cells of the same and between different types of cells. [20] Glycogen release in astrocytes can isolate excessive release of extracellular $\mathrm{K}+$ from the nerve. [21] The expression and activity of enzymes involved in glycogen metabolism have been shown to be induced by the vasoconstrictor endothelin- 1 in rats with ischemic stroke. At 6 and $24 \mathrm{~h}$, the level of glycogen in the ipsilateral cerebral hemisphere was elevated relative to the contralateral cerebral hemisphere ( $25 \%$ and $39 \%$, respectively), and there was a similarity in glycogen synthase activity and glycogen branching enzyme expression between the ipsilateral, contralateral and pseudocontrol hemispheres. The activity of rate-limiting enzyme involved in glycogen decomposition and glycogen phosphorylase decreased by $58 \% 24 \mathrm{~h}$ after stroke, and the activity of camp-dependent protein kinase A (PKA) was reduced by $48 \%$ in the same way. At $24 \mathrm{~h}$ after stroke, the rate of glycogen debranching enzyme expression was reduced by $77 \%$ and $72 \%$ at the protein and mRNA levels, respectively. [22] Hypoxia and inhibition of PKA activity significantly decreased glycogen phosphorylase activity and increased glycogen accumulation but did not change glycogen synthase activity. In addition, elevated glycogen levels provide metabolic support for astrocytes during periods of hypoxia. [22] During ischemic stroke, mitochondria in astrocytes can store a large amount of glycogen. Glycogen is more resilient and adaptable in a hypoxic environment to provide energy support for damaged neurons through their own biological systems and changes in mitochondrial dynamics. At the same time, as a bridge between the neural network and the vascular network, AS can regulate cerebral blood flow by contacting the arterioles and capillaries of the central nerve system to affect the diameter of blood vessels. [23]

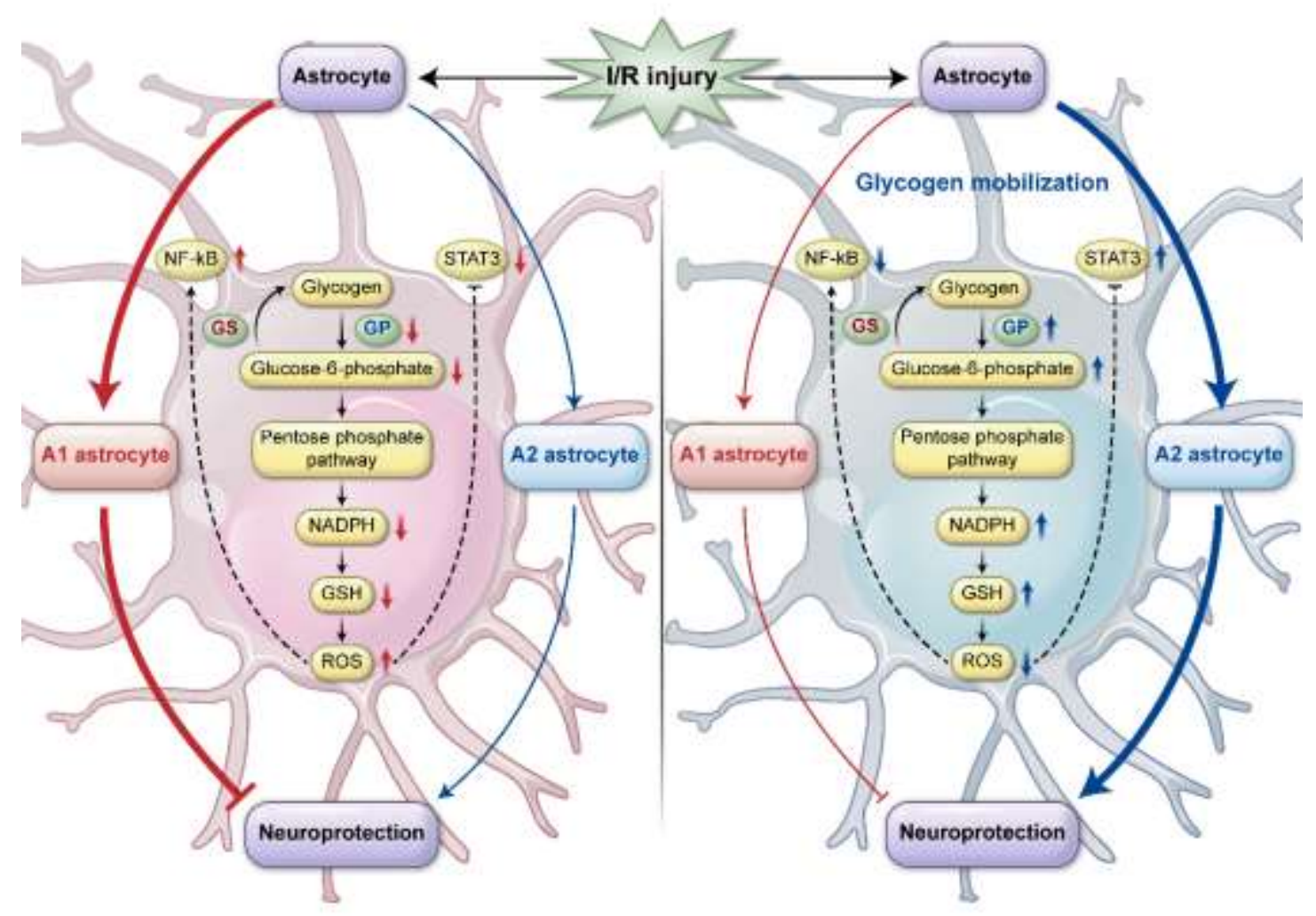

Figure 1: Schematic diagram depicting the mechanism underlying glycogen mobilization-mediated neuroprotection against reperfusion injury after ischemic stroke.

\section{Glycogen metabolism dysfunction in I/R}

Ischemia-reperfusion is designed to save penumbra tissue and reduce infarct size. The deficiency and restoration of blood flow disrupt the balance of brain energy and lead to injury during ischemic reperfusion. The common symptoms are glutamate poisoning, oxygen free radical overload, inflammation, and calcium overload. Recanalization of obstructed energy metabolism in the bloodstream can aggravate ischemia reperfusion injury. The loss of blood disrupts the brain's energy balance. Brain glycogen, which is rapidly decomposing, provides extra energy for the brain during blood vessel blockage. After glycogen breaks down, glucose-6-phosphate can be generated through glycolysis to generate lactic acid for aerobic oxidation. On the other hand, after gluconeogenesis, glucose reduces the amount of oxygen concentration in cells by NADPH and glutathione generated through pentose phosphate. [24] Cerebral ischemia leads to A1 (injury)/A2 (protection) differentiation of astrocytes. [8] NF-kb is a key molecule of A1; likewise, STAT3 is a key molecule of A2. Peripheral studies suggest that ROS can activate NF-KB and inhibit STAT3. Glycogen catabolism, mediated by the PKA-PHK-GP signaling pathway, is the main cause of glycogen 
accumulation in reperfusion and promoting the decomposition of glycogen can play a protective role. Post ischemia-reperfusion injury can inhibit A1 formation and elevate A2 activation. [8] (Figure 1).

\section{Glycogen metabolism is a promising therapeutic target for alleviating cerebral reperfusion injury}

Glycogen mobilization could reduce ROS levels and further inhibit the NF-KB signaling circuit and activate the STAT3 signaling circuit to play a neuroprotective role. [25] Astrocytes fill up the space of damaged nerve cells in the central nervous system to form nerve scarring with keratin and possibly aiding in nerve repair, and gene ablation studies have shown that astrocytes do play an integral role in regeneration. [26]

Immunofluorescence staining of glycogen phosphorylase is a key enzyme of astrocyte-specific glycogen catabolism. Immunofluorescence staining confirms that glycogen phosphorylase content is increased in overexpressed gene mice with significantly reduced infarction, and significantly improved limb sensory and motor functions. [27]

There is a protective effect of elevated glucose transporter (GLUT) activity on cells during ischemic energy depletion. In addition, under physiological conditions, cultured astrocytes express GLUT1 and GLUT3 at low levels. Under physiological conditions, cultured astrocytes promoted the percent conversion of the transcription factor nuclear factorJB (NF-JB) and increased GLUT3 expression under ischemic stress. Astrocyte GLUT3 improves resistance in response to fatal ischemic stress by increasing intracellular glucose storage during reperfusion. [28] Under pathological conditions, glycogen levels and metabolism could be influenced in cancer studies (gastric adenocarcinoma) and neurological diseases (Alzheimer's disease). [29-32]

Astrocyte glycogenolysis and lactic acid play a key role in memory formation, and astrocytic B2 adrenergic receptor and lactate can enhance memory. Glycogen decomposition plays an important role in memory and astrocyte-neuronal metabolic coupling primarily at the peak of brain development could meet energy requirements. [33]

Obviously, the aggregate formation of glycogen is the cause of epilepsy. In epilepsy studies with Lafora disease as an example, in an LD animal model, glycogen aggregates gathered into polyglucosan bodies, which are called Lafora bodies (LBs), suggesting that the elimination of glycogen synthase (GS) prevented the formation of LBs and the development of LDs. This also suggests that glycogen accumulation is the basis of the disease and related symptoms, further revealing the relationship between glycogen accumulation and seizures. [34] Meanwhile, this confirms that glycogen deficiency can reduce the occurrence of epilepsy. [34]

In ischemic rats, the deletion of CDK5 in astrocytes resulted in the recovery of motor function, the increase in perivascular endogenous astrocyte branches and endothelial PECAM1 and proliferation markers, and it also restored the blood-brain barrier and secretion of BDNF through endogenous astrocytes in ischemic rats. [35]

Ganglioside GM1 can increase glucose intake and mobilize glycogen storage. Additionally, it can stimulate the secretion of lactic acid from astrocytes and enhance the gene expression of PTG, hexokinase, $\mathrm{Na}+\mathrm{K}+$ ATPASE $\alpha 2$ subunit, pyruvate dehydrogenase and transaldolase. The metabolic and neuroprotective roles of astrocytes were further identified and confirmed in brain diseases, such as spinal cord injury, Huntington's disease and Parkinson's disease. [36]

In chronic pain, lactic acid promotes astrocyte proliferation and reactive astrocyte proliferation through a common intracellular signaling pathway that promotes synaptic activity in the central nervous system, possibly due to the activity-dependent release of astrocytes to maintain continuously elevated extracellular lactic acid levels. Lactic acid has lasting effects on astrocytes and neurons mediated by extracellular receptor binding. [37] Apparently, the pathology of the central nervous system is often associated with reactive astrocyte hyperplasia accompanied by changes in the release of some neuroregulatory substances. Additionally, astrocytes are involved. [38]

\section{Conclusion}

In general, astrocyte glycogen is dynamic and active, and glycogen mobilization plays an integral role in neuronal development. In addition to changes in the physiological environment, for example, $I / R$, the dynamic changes, decomposition, accumulation and alienation of astrocyte glycogen are not negligible. At the same time, astrocyte glycogen is regarded as a new therapeutic target and has attracted much attention in the fields of nervous system diseases, cancer, and pain. The role and mechanism of astrocyte glycogen mobilization in other fields deserves further investigation and attention.

\section{Funding}

This study was financially supported by the National Natural Science Foundation of China (nos. 82001384 and 81901079).

\section{Acknowledgement}

Not applicable.

\section{Data Availability Statement}

The datasets generated during the current study are available from the corresponding author upon reasonable request.

\section{Conflicts of Interest}

The authors declare no conflict of interest.

\section{References}

1. Qu, X., Wu, S., Gao, J., Qin, Z., Zhou, Z., \& Liu, J. (2021). Weighted gene co expression network analysis (WGCNA) with key pathways and hub-genes related to micro RNAs in ischemic stroke. IET systems biology, 15(3), 93-100.

2. Nan Zhao. (2018) Effects of lipocalin 2 on the functional polarization of astrocytes induced by cerebral ischemia in mice

3. Scemes, E., Suadicani, S. O., \& Spray, D. C. (2000). Intercellular communication in spinal cord astrocytes: fine tuning between gap junctions and P2 nucleotide receptors in calcium wave propagation. The Journal of neuroscience: the official journal of the Society for Neuroscience, 20(4), 14351445.

4. Nagy J I \& Rash J E.(2000). Connexins and gap junctions of astrocytes and oligodendrocytes in the CNS. Brain research. Brain research reviews (1),

5. Lee JC, Chen BH, Cho JH et al (2015) Changes in the expression of DNA-binding/differentiation protein inhibitors in neurons and glial cells of the gerbil hippocampus following transient global cerebral ischemia. Mol Med Rep 11:247724853.

6. Araque A, Parpura V, Sanzgiri RP, Haydon PG (May 1999)."Tripartite synapses: glia, the unacknowledged partner". Trends in Neurosciences. 22 (5):208-215.

7. McDougal DH, Viard E, Hermann GE, Rogers RC (April 2013). "Astrocytes in the hindbrain detect glucoprivation and 
regulate gastric motility". Autonomic Neuroscience. 175 (1-2): 61-69.

8. Cai Y, Guo H, Fan Z, Zhang X, Wu D, Tang W, Gu T, Wang S, Yin A, Tao L, Ji X, Dong H, Li Y, Xiong L. Glycogenolysis Is Crucial for Astrocytic Glycogen Accumulation and Brain Damage after Reperfusion in Ischemic Stroke. iScience. 2020 May 6;23(5):101136.. Epub ahead of print.PMID: 32446205; PMCID: PMC7240195.

9. Obel, L. F., Müller, M. S., Walls, A. B., Sickmann, H. M., Bak, L. K., Waagepetersen, H. S., \& Schousboe, A. (2012). Brain glycogen-new perspectives on its metabolic function and regulation at the subcellular level. Frontiers in neuroenergetics, 4,3 .

10. DiNuzzo, M., Mangia, S., Maraviglia, B., \& Giove, F. (2010). Glycogenolysis in astrocytes supports blood-borne glucose channeling not glycogen-derived lactate shuttling to neurons: evidence from mathematical modeling. Journal of cerebral blood flow and metabolism : official journal of the International Society of Cerebral Blood Flow and Metabolism, 30(12), 18951904.

11. DiNuzzo, M., Mangia, S., Maraviglia, B., \& Giove, F. (2010). Changes in glucose uptake rather than lactate shuttle take center stage in subserving neuroenergetics: evidence from mathematical modeling. Journal of cerebral blood flow and metabolism : official journal of the International Society of Cerebral Blood Flow and Metabolism, 30(3), 586-602.

12. Dinuzzo M, Mangia S, Maraviglia B, Giove F. The role of astrocytic glycogen in supporting the energetics of neuronal activity. Neurochem Res. 2012 Nov;37(11):2432-2438. doi: 10.1007/s11064-012-0802-5. Epub 2012 May 22. PMID: 22614927; PMCID: PMC4062197.

13. Rossi DJ, Brady JD, Mohr C. Astrocyte metabolism and signaling during brain ischemia. Nat Neurosci. 2007 Nov;10(11):1377-1386. doi: 10.1038/nn2004. PMID: 17965658.

14. Brown AM, Sickmann HM, Fosgerau K, Lund TM, Schousboe A, Waagepetersen HS, Ransom BR. Astrocyte glycogen metabolism is required for neural activity during aglycemia or intense stimulation in mouse white matter. J Neurosci Res. 2005 Jan 1-15;79(1-2):74-80.. PMID: 15578727.

15. Hertz L, Gibbs ME. What learning in day-old chickens can teach a neurochemist: focus on astrocyte metabolism. J Neurochem. 2009 May;109 Suppl 1:10-16. PMID: 19393003.

16. Hertz, L., O'Dowd, B. S., Ng, K. T., \& Gibbs, M. E. (2003). Reciprocal changes in forebrain contents of glycogen and of glutamate/glutamine during early memory consolidation in the day-old chick. Brain research, 994(2), 226-233.

17. Lee CH, Ahn JH, Won MH (2015) New expression of 5-HT1A receptor in astrocytes in the gerbil hippocampal CA1 region following transient global cerebral ischemia. Neurol Sci 36:383-389.

18. Duncan PW, Zorowitz R, Bates B, Choi JY, Glasberg JJ, Graham GD, Katz RC, Lamberty K, Reker D. Management of Adult Stroke Rehabilitation Care: a clinical practice guideline. Stroke. 2005 Sep;36(9):e100-143. PMID: 16120836.

19. Park OK, Lee CH, Hwang IK et al (2010) Effects of repeated restraint stress on platelet endothelial cell adhesion molecule-1 immunoreactivity and protein levels in the gerbil hippocampus after transient cerebral ischemia. Anat Cell Biol 43:54-63

20. Li Y, Liu Z, Xin H, Chopp M. The role of astrocytes in mediating exogenous cell-based restorative therapy for stroke. Glia. 2014 Jan;62(1):1-16. doi:10.1002/glia.22585. Epub 2013 Nov 4. PMID: 24272702; PMCID: PMC3947888.
21. Shulman, R. G., Hyder, F., \& Rothman, D. L. (2001). Cerebral energetics and the glycogen shunt: neurochemical basis of functional imaging. Proceedings of the National Academy of Sciences of the United States of America, 98(11), 6417-6422.

22. Hossain MI, Roulston CL, Stapleton DI. Molecular basis of impaired glycogen metabolism during ischemic stroke and hypoxia. PLoS One.2014 May 23;9(5):e97570.. Erratum in: PLoS One. 2014;9(8):e106173. PMID: 24858129; PMCID: PMC4032261.

23. Xiaomei Su, Danshen Zhang, Chinese Journal of Pharmacology and Toxicology. (09,2021) Astrocytes save ischemic stroke. Chinese Journal of Pharmacology and Toxicology, 2021,09:671

24. Ilpo Huhtaniemi , Luciano Martini (2018). Glucose Metabolism and Hormonal Regulation, Encyclopedia of Endocrine Diseases (Second Edition),Academic Press,(1)87-94, -3.03816-2.

25. Guo H, Fan Z, Wang S, Ma L, Wang J, Yu D, Zhang Z, Wu L, Peng Z, Liu W, Hou W, Cai Y. Astrocytic A1/A2 paradigm participates in glycogen mobilization mediated neuroprotection on reperfusion injury after ischemic stroke. J Neuroinflammation. 2021 Oct 13;18(1):230. PMID:34645472; PMCID: PMC8513339.

26. Liddelow SA, Guttenplan KA, Clarke LE, Bennett FC, Bohlen CJ, Schirmer L, Bennett ML, Münch AE, Chung WS, Peterson TC, Wilton DK, Frouin A, Napier BA, Panicker N, Kumar M, Buckwalter MS, Rowitch DH, Dawson VL, Dawson TM, Stevens B, Barres BA (January 2017). "Neurotoxic reactive astrocytes are induced by activated microglia". Nature. 541 (7638): 481-487. Bibcode:2017Natur.541..481L. PMC 5404890. PMID 28099414.

27. Mo W, Li L, Lin W, Sheng-xia Zhang, Hai-yun G \& Yan-hui C (2020)" Experimental Study of Glycogen Mobilization Mediated Neuroprotection on Cerebral Ischemia/reperfusion injury". Progress in Modern Biomedicine. 2020,20:3822-3826. 2020.20.004

28. Iwabuchi S, Kawahara K. Inducible astrocytic glucose transporter-3 contributes to the enhanced storage of intracellular glycogen during reperfusion after ischemia. Neurochem Int. 2011 Aug;59(2):319-325. Epub 2011 Jun 15. PMID: 21703319.

29. Govender, D., Ramdial, P. K., Clarke, B., \& Chetty, R. (2004). Clear cell (glycogen-rich) gastric adenocarcinoma. Annals of diagnostic pathology, 8(2), 69-73.

30. Brucklacher, R. M., Vannucci, R. C., \& Vannucci, S. J. (2002). Hypoxic preconditioning increases brain glycogen and delays energy depletion from hypoxia-ischemia in the immature rat. Developmental neuroscience, 24(5), 411-417.

31. Lau, X., Zhang, Y., Kelly, D.J. et al. (2013). Attenuation of Armanni-Ebstein lesions in a rat model of diabetes by a new anti-fibrotic, anti-inflammatory agent, FT011. Diabetologia 56, 675-679

32. Sabino Vesce, Daniela Rossi, Liliana Brambilla, Andrea Volterra,( 2007).Glutamate Release from Astrocytes in Physiological Conditions and in Neurodegenerative Disorders Characterized by Neuroinflammation,International Review of Neurobiology, 57-71,

33. Alberini, C. M., Cruz, E., Descalzi, G., Bessières, B., \& Gao, V. (2018). Astrocyte glycogen and lactate: New insights into learning and memory mechanisms. Glia, 66(6), 1244-1262.

34. Duran, J., Gruart, A., López-Ramos, J. C., Delgado-García, J. M., \& Guinovart, J. J. (2019). Glycogen in Astrocytes and Neurons: Physiological and Pathological Aspects. Advances in neurobiology, 23, 311-329. 
35. Becerra-Calixto, A., Posada-Duque, R., \& Cardona-Gómez, G. P. (2018). Recovery of Neurovascular Unit Integrity by CDK5KD Astrocyte Transplantation in a Global Cerebral Ischemia Model. Molecular neurobiology, 55(11), 8563-8585.

36. Finsterwald, C., Dias, S., Magistretti, P. J., \& Lengacher, S. (2021). Ganglioside GM1 Targets Astrocytes to Stimulate Cerebral Energy Metabolism. Frontiers in pharmacology, 12, 653842 .
37. Lampe, K. J., Namba, R. M., Silverman, T. R., Bjugstad, K. B., \& Mahoney, M. J. (2009). Impact of lactic acid on cell proliferation and free radical-induced cell death in monolayer cultures of neural precursor cells. Biotechnology and bioengineering, 103(6), 1214-1223. 22352

38. Tang, J., Bair, M., \& Descalzi, G. (2021). Reactive Astrocytes: Critical Players in the Development of Chronic Pain. Frontiers in psychiatry, 12, 682056.
This work is licensed under Creative Commons Attribution 4.0 License
Submit Manuscript
To Submit Your Article Click Here:

DOI: $10.31579 / 2578-8868 / 228$
Ready to submit your research? Choose Auctores and benefit from:

$>$ fast, convenient online submission

$>$ rigorous peer review by experienced research in your field

$>$ rapid publication on acceptance

$>$ authors retain copyrights

$>$ unique DOI for all articles

$>$ immediate, unrestricted online access

At Auctores, research is always in progress.

Learn more https://auctoresonline.org/journals/neuroscience-and-neurologicalsurgery 